EUROPEAN ORGANIZATION FOR NUCLEAR RESEARCH

CERN-EP/98-61

12 May 1998

\title{
STUDY OF THE EVOLUTION OF THE ACTIVE VOLUME IN IRRADIATED SILICON DETECTORS
}

\author{
G. Casse*), E. Grigoriev, F. Lemeilleur, M. Glaser \\ CERN, Geneva, Switzerland
}

ROSE Collaboration - RD48

\begin{abstract}
Red $(670 \mathrm{~nm})$ LED light was used to study the charge collection properties of non-irradiated and irradiated n-type silicon detectors. The advantages of red LED, compared to low-range alpha particles, are the availability of an external trigger, and a very shallow distribution of the created electron-hole pairs $(<10 \mu \mathrm{m})$. These features, combined with the use of a fast current amplifier and a $2.5 \mathrm{Gs} / \mathrm{s}$ sampling oscilloscope, allow the electric field evolution in irradiated detectors to be studied. Evidence of a sensitive region on both sides of the detector was observed. The model of the diode depletion volume from the $\mathrm{n}^{+}$junction side after conduction-type inversion is discussed, and the electric field distribution in the inverted detector is presented. A first evaluation of the strength of the electric field in the undepleted bulk of the detector is proposed.
\end{abstract}

Presented at the 2nd International Conference on Radiation Effects on Semiconductor Materiels, Detectors and Devices, Firenze 4-6 March 1998

*) ASP: Associazione per lo sviluppo Scientifico del Piemonte 


\section{INTRODUCTION}

In this study, several similar n-type silicon diodes, with an initial $2.2 \mathrm{k} \Omega \mathrm{cm}$ resistivity and a thickness of $\approx 300 \mu \mathrm{m}$, were irradiated with fluences ranging from $1 \times 10^{12} \mathrm{~cm}^{-2}$ up to $2.9 \times 10^{14} \mathrm{~cm}^{-2}$, as shown in Table 1. The irradiation was performed in the $24 \mathrm{GeV} / \mathrm{c}$ proton beam at the CERN PS. In order to allow the injection of light into the detector volume from both sides, the silicon diodes have a window in the metallized front $\mathrm{p}^{+}-n$ junction, and an aluminium grid, leaving $100 \mu \mathrm{m}$ width non-metallized windows in the rear contact. The light injection is performed using a LED driven by an external pulse generator which also provides the trigger to the oscilloscope for recording the pulse. The LED pulse shape is shown in Fig. 1, as recorded by a photomultiplier. The FWHM of the LED pulse is $\approx 15 \mathrm{~ns}$. The LED is pig-tailed with a multimode optical fibre of $0.5 \mathrm{~mm}$ diameter which illuminates the detector through the front window or the rear grid. The detector signal is amplified by two fast current amplifiers (Phillips Scientific model 6954) with total gain of 1000. An averaging of the signal is performed with $\approx$ 400 triggers. The signal pulse shape and the integral of the signal with time were recorded with a $300 \mathrm{MHz}$ Lecroy 9361 oscilloscope. The charge collection efficiency (CCE) as a function of the bias is normalized to the maximum value $Q_{\mathrm{M}}$, defined as the averaged value of the charge collected over a few points with an operating bias above the full depletion voltage $\left(V_{\mathrm{FD}}\right)$. This normalization allows a better comparison between the CCE curves for detectors irradiated with different fluences.

Table 1

\begin{tabular}{|c|c|c|c|c|c|}
\hline Detector name & Fluence $\left[\mathrm{cm}^{-2}\right]$ & Detector name & Fluence & Detector name & Fluence \\
\hline P189-S-A1-18 & Non irradiated & P12-S-A3-11 & $5.2 \times 10^{13}$ & P12-S-A3-16 & $1.7 \times 10^{14}$ \\
P189-S-A1-17 & $1 \times 10^{12}$ & P88-S-A1-19 & $1 \times 10^{14}$ & P88-S-A1-17 & $2.9 \times 10^{14}$ \\
P88-S-A1-14 & $1.5 \times 10^{13}$ & P189-S-A1-22 & $1 \times 10^{14}$ & P189-S-A1-21 & $2.9 \times 10^{14}$ \\
\hline
\end{tabular}

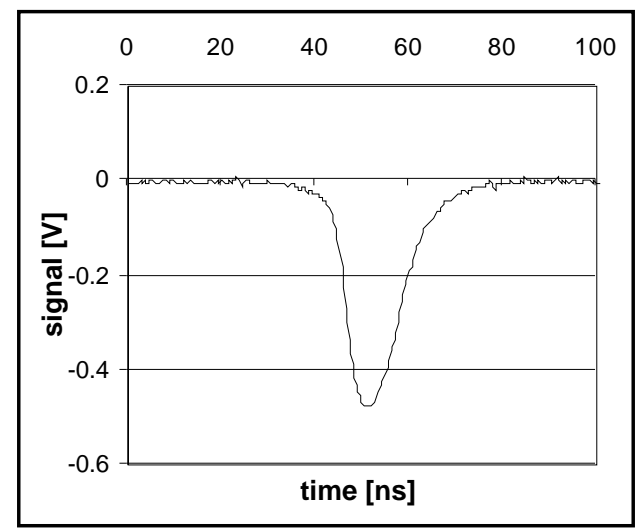

Fig. 1: LED light pulse recorded with a photomultiplier 


\section{CHARGE COLLECTION BEFORE IRRADIATION}

The absorption coefficient of the red LED light with a wavelength of $670 \pm 50 \mathrm{~nm}$ is $\approx 3 \times 10^{3} \mathrm{~cm}^{-1} .90 \%$ of the photons are absorbed within $7 \mu \mathrm{m}$ depth of the silicon (Fig. 2). This depletion depth corresponds to reverse bias voltages ranging from $\approx 0.2 \mathrm{~V}$ for non-irradiated detectors to $\approx 0.4 \mathrm{~V}$ for the highest irradiated detectors. As these values are lower than the builtin voltage $(\approx 0.6 \mathrm{~V})$, it can be assumed that all the charge is generated in a depleted layer of the detector when the light illuminates the junction side. This very shallow distribution allows the electron signal (for front illumination) and the hole signal (for rear illumination) to be studied.

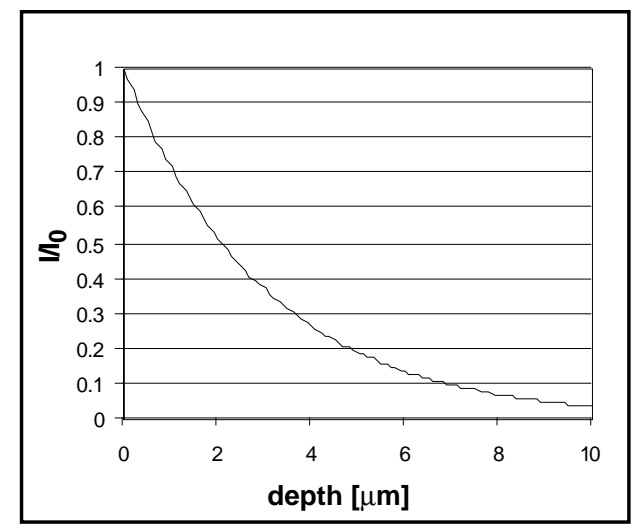

Fig. 2: Distribution of generated electron-hole pairs in silicon illuminated with light of wavelength $670 \mathrm{~nm}$

The depletion region in a non-irradiated detector develops from the front $\mathrm{p}^{+}-n$ junction. When the charge is injected through the front contact, there is complete charge collection even without any external reverse bias, due to the depleted region created by the built-in voltage, as shown in Fig. 3. Figure 4a shows the pulse shape for different values of the bias with the nonirradiated detector. The charge collection without any external reverse bias takes a very long time (> $200 \mathrm{~ns}$ ). When an external reverse bias is applied, the collection is faster due to the increased velocity of the moving charge, proportional to the strength of the electric field. The peak time of the signal is centred at $\approx 12 \mathrm{~ns}$ (peak time - trigger time) and it is only slightly, or not at all, influenced by increasing the bias. The signal is caused by the electrons moving in the electric field of the diode [1]. Its maximum amplitude coincides with the maximum of the electric field, which occurs at the junction position and decreases inside the detector. The higher velocity of the electron, responsible for the peak time, is at the beginning of the motion $[2,3]$.

When the LED illuminates the rear side of an undepleted detector, the charge is generated in a non-sensitive region and no current pulse is induced on the diode electrodes. The pulse appears when the depletion region, developing from the junction (front) side, reaches the layer where the charge is deposited. The charge collection reaches maximum efficiency when the diode is fully depleted, as shown in Fig. 3. This allows the full depletion voltage $\left(V_{\mathrm{FD}}\right)$ of the detector to be estimated. Figure 3 also shows the $V_{\text {FD }}$ evaluated by the capacitance-voltage (CV) method. It is evident that complete charge collection takes place at a bias corresponding to the minimum capacitance. It can also be remarked that a partial charge collection starts well before full depletion is achieved. This is due to the charge diffusing in the non-depleted bulk towards the depleted region. In this case the collection time is large compared to the collection time of 
the charge produced in the electric field region, as shown in Fig. 4b. Figure 5 shows the dependence of the peaking time as a function of $V^{1 / 2}$ for rear illumination. The decrease of the peak time is attributed mainly to the reduction in the diffusion path for bias values below $V_{\mathrm{FD}}$, and to the earlier arrival of the holes in the maximum field region for bias voltages higher than $V_{\mathrm{FD}}$. With rear injection, the holes move towards the increasing electric field. By increasing the bias, the increased charge velocity allows the charge to reach the maximum field region in a shorter time. With front injection, on the contrary, the peak position does not depend on the reverse bias voltage. The behaviour of the peak position as a function of bias can therefore provide useful information about the electric field shape.

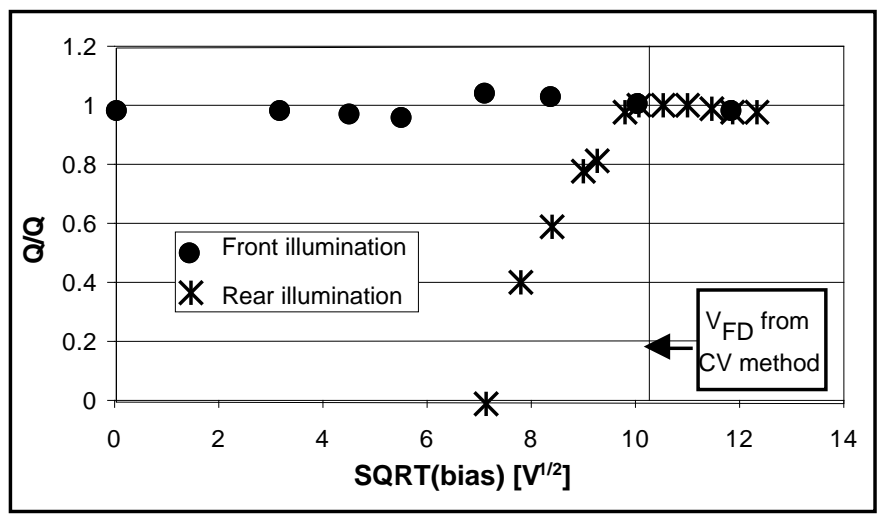

Fig. 3: $\mathrm{CCE}$ as a function of $V^{1 / 2}$ for a non-irradiated diode

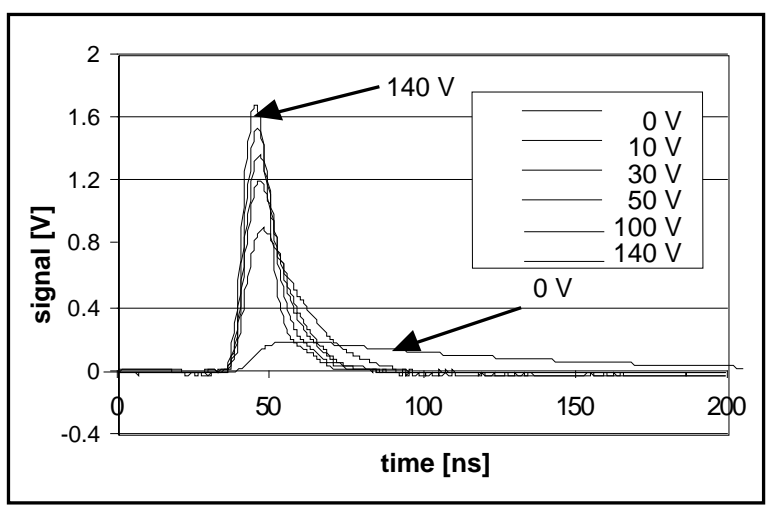

a)

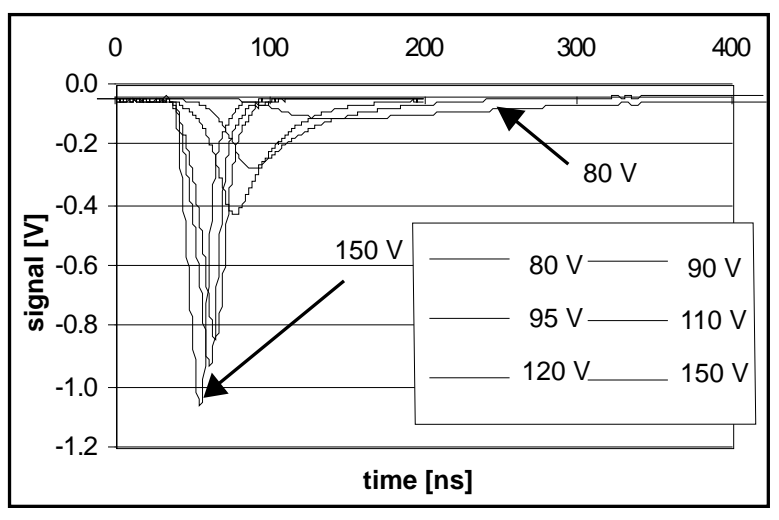

b)

Fig. 4: Signal pulse shape for a non-irradiated diode. (a) front illumination (b) Rear illumination. $V_{\mathrm{FD}}=105 \mathrm{~V}$ 


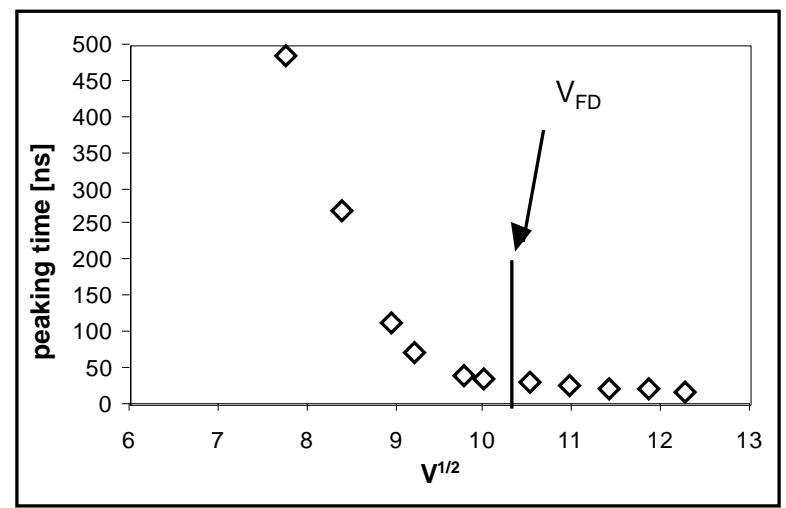

Fig. 5: Peaking time (peak time - trigger time) as a function of $V^{1 / 2}$ for rear injection for a non-irradiated diode

\section{CHARGE COLLECTION FOR INVERTED DIODES}

It is well established that the effective doping concentration $\left(N_{\text {eff }}\right)$ in an n-type silicon detector decreases during hadron irradiation until the type inversion fluence is reached [4]. The electrical conductivity then converts from n-type to p-type. Further irradiation makes the detector more effectively p-type, causing the linear increase of $N_{\text {eff }}$ as a function of fluence. After inversion, a simple model describes the detector as a p-type silicon diode. The junction therefore moves to the rear side of the detector and the depletion region develops from the new $\mathrm{n}^{+}-\mathrm{p}-$ effective junction. This model predicts no charge collection in an inverted detector illuminated from the front side with red light, until the depletion region (developing from the rear side) reaches the penetration depth of the injected light. The inversion fluence for the detectors used in this work was $\approx 5 \times 10^{13} \mathrm{~cm}^{-2}$.

The charge collection for inverted diodes illuminated through the rear side is linearly dependent on the square root of bias even if the charge is entirely deposited in the depleted volume of the detector, as shown in Fig. 6a. Before irradiation, a non-depleted detector is equivalent to a thinner fully-depleted diode of thickness equal to the depletion layer, because the electrical contact is brought to the boundary of the depletion layer by the conductive undepleted bulk. In this case, complete charge collection takes place. To explain the different behaviour of the irradiated diodes, it has been proposed that the undepleted bulk is resistive for the fast currents generated by ionization [5]. The moving charge induces a signal until it reaches the boundary of the sensitive bulk. The collection time, $t$, can be expressed as

$$
t_{\mathrm{c}}=\frac{x_{\mathrm{d}}}{v_{\text {average }}}=\frac{x_{\mathrm{d}}}{\frac{1}{x_{\mathrm{d}}} \int_{0}^{x_{d}} \mu E(x) \mathrm{d} x}=\frac{x_{\mathrm{d}}}{\frac{1}{x_{\mathrm{d}}} \int_{0}^{x_{\mathrm{d}}} \frac{\mu \mathrm{dV}(x)}{\mathrm{d} x} \mathrm{~d} x}=\frac{x_{\mathrm{d}}^{2}}{\mu V_{\mathrm{bias}}},
$$

where $x_{\mathrm{d}}$ is the drift distance and the mobility $\mu$ is considered to be constant. The quantity $x_{\mathrm{d}}$ is proportional to the square root of bias and therefore $t_{\mathrm{c}}$ is constant. The charge collected for two different biases $V_{1}$ and $V_{2}$ can be expressed using Eq. (A.10).

$$
Q_{\mathrm{e}}\left(V_{1,2}\right)=\frac{n_{0} x_{\mathrm{d} 1,2}}{w}\left\lfloor\exp \left(-\frac{\mu_{\mathrm{e}} q N e_{\mathrm{ff}} t_{\mathrm{c}}}{\varepsilon_{S i}}\right)-1\right\rceil
$$


$x_{\mathrm{d} 1,2}$ being the depletion depths. The difference between the charge collected for the two biases, where $V_{1}<V_{2}<V_{\mathrm{FD}}$, is

$Q_{e}\left(V_{2}\right)-Q_{e}\left(V_{1}\right)=\frac{n_{0}}{w}\left\lceil\exp \left(-\frac{\mu_{e} q N e_{\mathrm{ff}} t_{\mathrm{c}}}{\varepsilon_{S i}}\right)-1\right\rceil\left(x_{\mathrm{d} 2}-x_{\mathrm{d} 1}\right) \propto x_{\mathrm{d} 2}-x_{\mathrm{d} 1}=\left(\frac{2 \varepsilon_{S i}}{q N_{\mathrm{eff}}}\right)^{\frac{1}{2}}\left(\sqrt{V_{2}-V_{1}}\right)$

$w$ being the detector thickness and $n_{0}$ the deposited charge.

The charge collection is then proportional to $V^{1 / 2}$, and for increasing irradiation levels after type inversion the CCE versus the square root of bias has a decreasing slope, because of the increase of $N_{\text {eff }}$ with irradiation. The CCE is linearly proportional to $V^{1 / 2}$ for rear illumination in inverted detectors. This indicates that the junction has moved from the front to the rear contact.

Figure $6 \mathrm{a}$ also shows that the CCE continues to increase after full depletion, as measured with the CV method. This can be caused by the trapping of charge carriers in an irradiated diode. The trapping effectiveness decreases with the carrier velocity. The amount of charge collected can be expressed, as a function of time, by

$$
n(t)=n_{0} e^{-\frac{t_{\mathrm{c}}}{\tau_{\mathrm{tr}}}}
$$

where $\tau_{\mathrm{tr}}$ is the trapping lifetime. The reduction of the charge collection below $V_{\mathrm{FD}}$ due to the trapping effect is constant, because of the independence of $t_{\mathrm{c}}$ on $V^{1 / 2}$ up to full depletion. Above $V_{\mathrm{FD}}$, the drift distance coincides with the detector thickness whilst the velocity increases with bias (until saturation). The subsequent reduction of $t_{\mathrm{c}}$ results in an improvement of the charge collection above $V_{\mathrm{FD}}$ according to Eq. (4).

The analysis of the pulse shapes in the inverted diodes as a function of bias confirms the migration of the junction from the front to the rear side. 


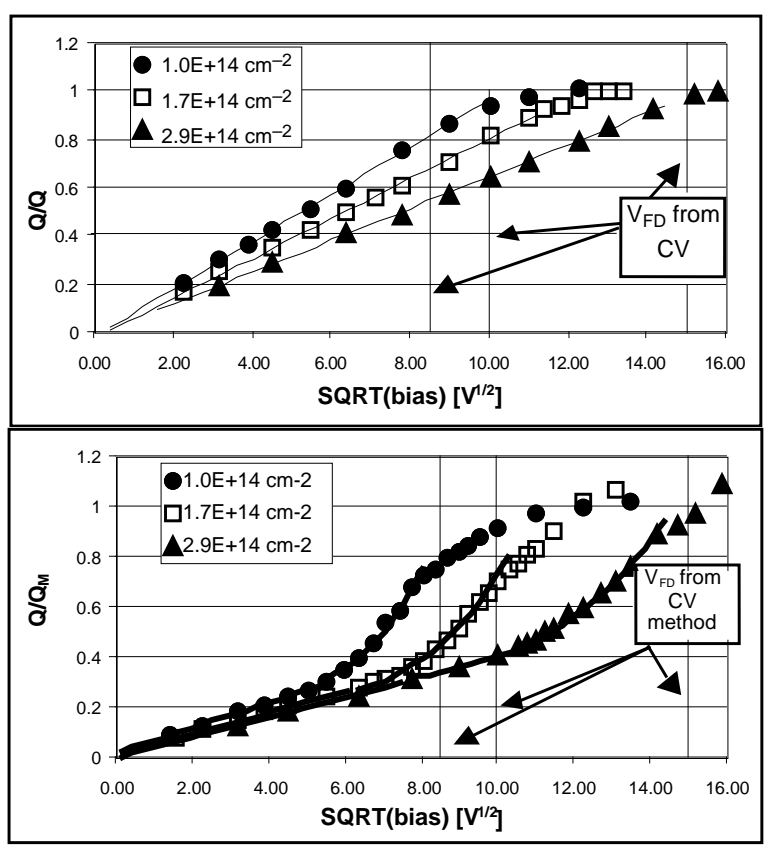

(a) (b)

Fig. 6: CCE as a function of $V^{1 / 2}$ of the inverted irradiated diode: (a) Rear illumination (b) Front illumination

When the light illuminates the rear side (Figs. $7 d, 8 d$ and 9d), the signal is visible from low- bias voltages, as expected from the injection in the electric field region. The peak time is centred at $\approx 14 \mathrm{~ns}$ and it does not evolve with increasing bias, indicating that the holes drift towards the cathode in the direction of the decreasing electric field.

After type inversion, according to the model of the inverted detector, the light injected from the front side should not induce any signal up to bias values close to $V_{\mathrm{FD}}$. On the other hand, charge collection takes place even at low biases, as can be seen in Fig. 6b. The CCE is proportional to the $V^{1 / 2}$ for low bias voltages. Figures $7(a, b, c), 8(a, b, c)$ and $9(a, b, c)$ show the pulse shape for different bias voltages for front illumination with increasing fluences, after type-inversion. A pulse appears from low biases, well below the $V_{\mathrm{FD}}$. The peak time of this pulse is $\approx 12 \mathrm{~ns}$ and it does not evolve with bias. The invariance of the peak time indicates that the charge is moving in a decreasing electric field. This fact excludes the possibility that the signal could be due to the diffusion of the charge carriers in a non-depleted bulk and indicates the presence of a depleted layer and an electric field next to the $\mathrm{p}^{+}$contact. This situation can be regarded as a double junction in inverted irradiated detectors.

A second peak appears when the bias is $\approx 35, \approx 50$ and $\approx 100 \mathrm{~V}$ for the detector irradiated to the fluence of $1 \times 10^{14} \mathrm{~cm}^{-2}, 1.7 \times 10^{14} \mathrm{~cm}^{-2}$ and $2.9 \times 10^{14} \mathrm{~cm}^{-2}$, respectively. The two peaks are well separated at the beginning. By increasing the bias, the time of the second peak decreases and the amount of charge carried by it increases. Eventually, the second peak becomes predominant and the two peaks cannot be separated anymore. 


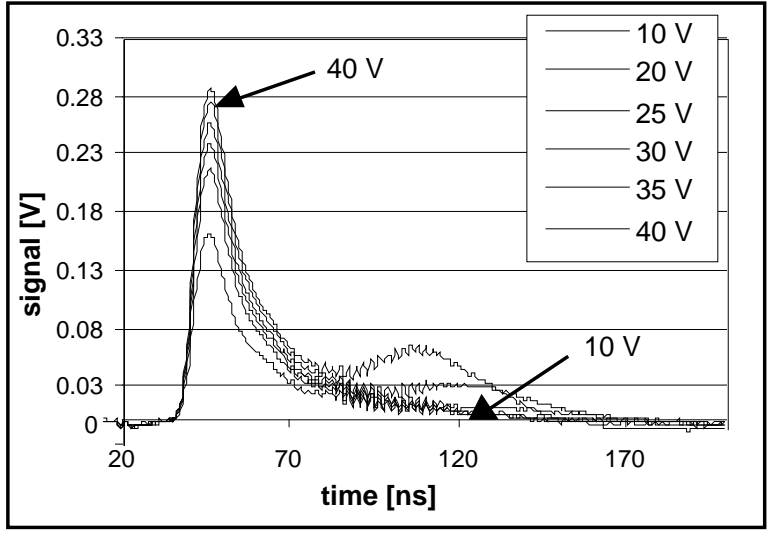

(a)

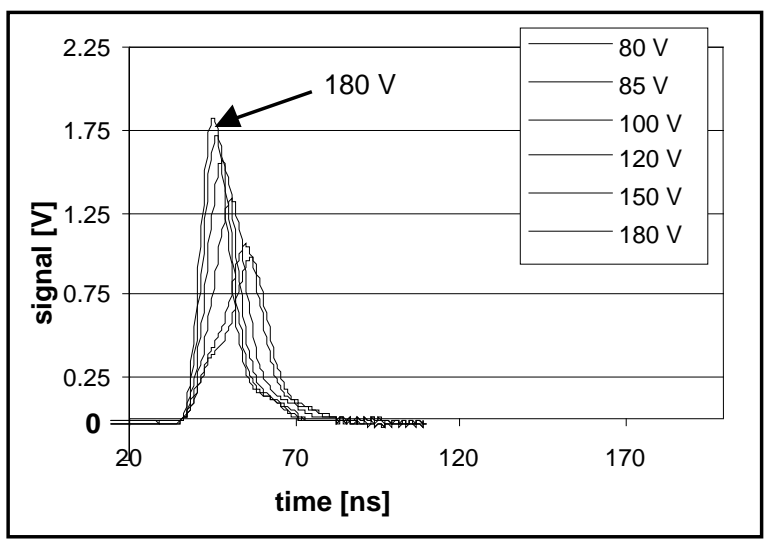

(c)

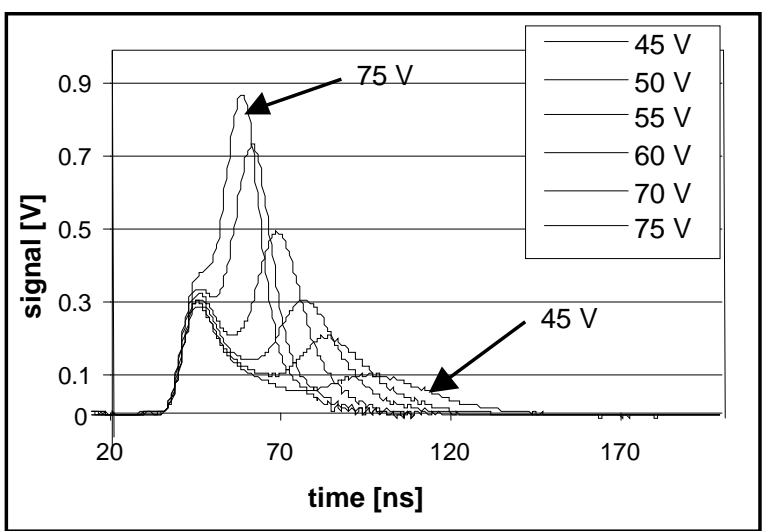

(b)

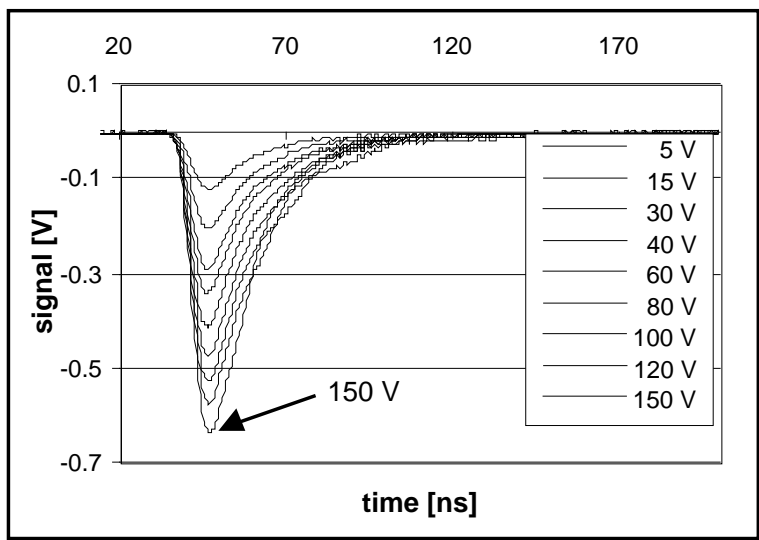

(d)

Fig. 7: Signal pulse shape for diode irradiated up to $1 \times 10^{14} \mathrm{~cm}^{-2}$ as a function of bias (a) (b) (c). Front illumination. $V_{\mathrm{FD}}=75 \mathrm{~V}$

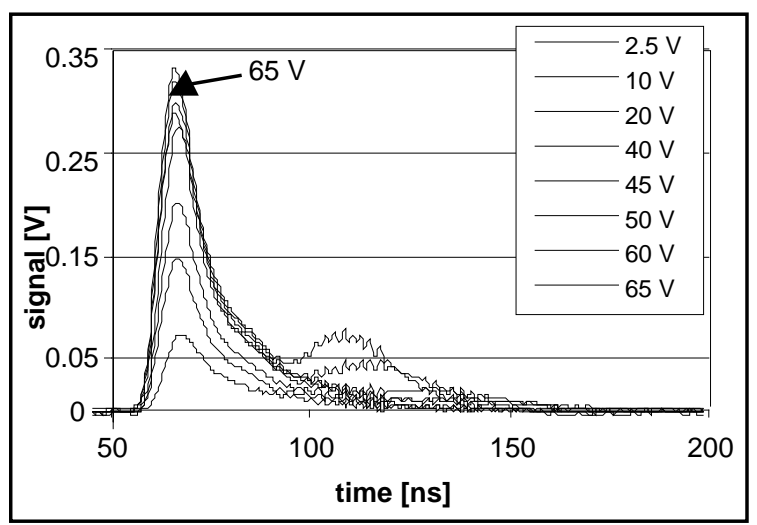

(a)

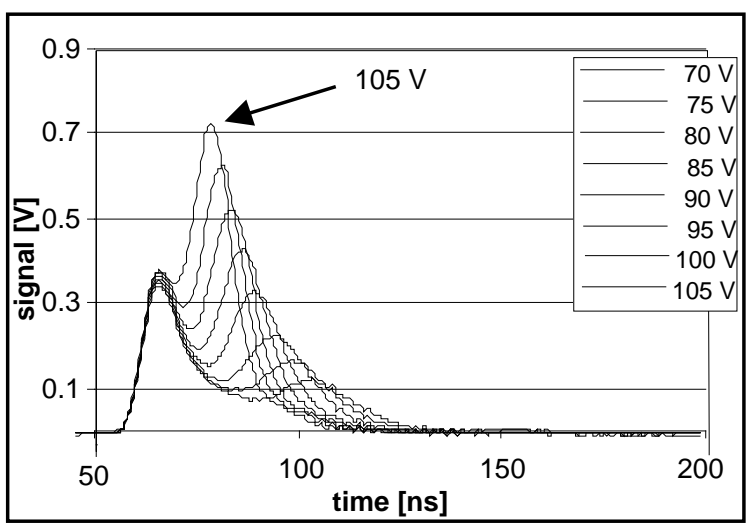

(b) 


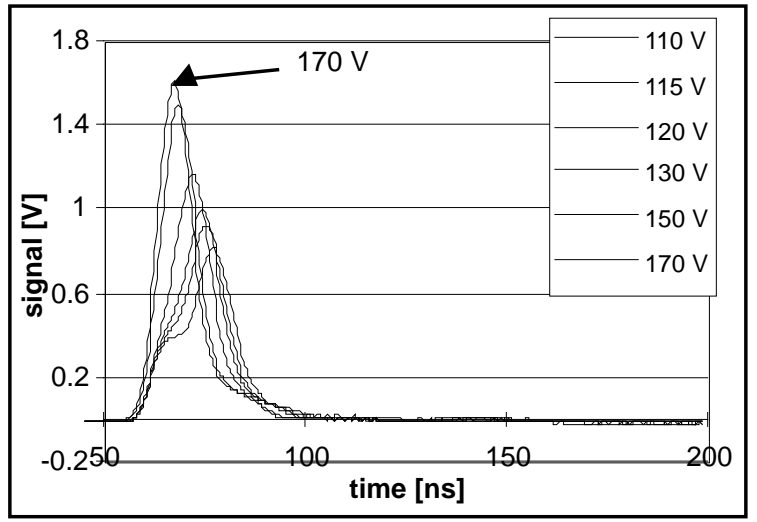

(c)

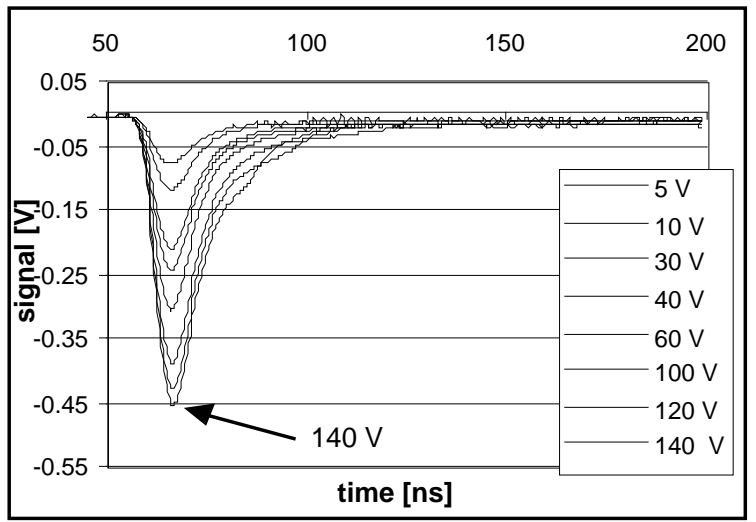

(d)

Fig. 8: Signal pulse shape for diode irradiated up to $1.7 \times 10^{14} \mathrm{~cm}^{-2}$ as a function of bias (a) (b) (c).

Front illumination. (d) Rear illumination. $V_{\mathrm{FD}}=108 \mathrm{~V}$

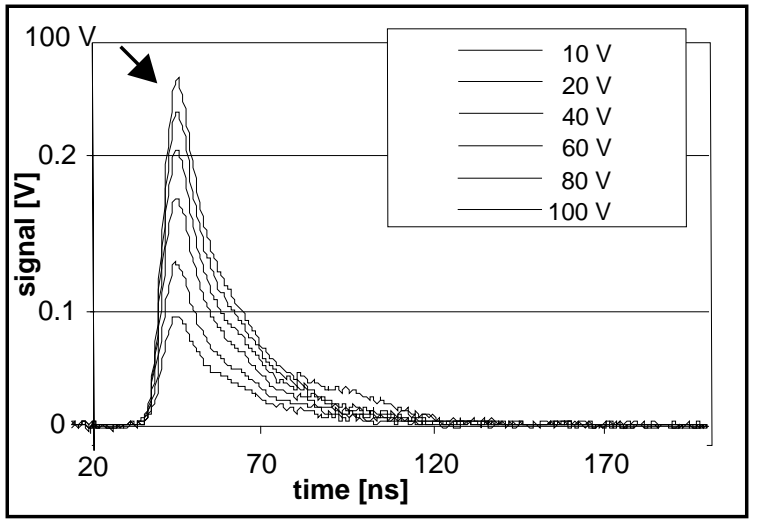

(a)

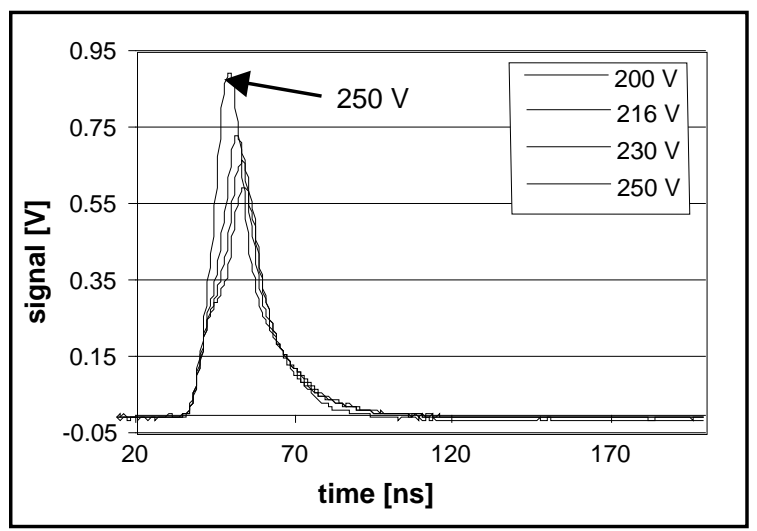

(c)

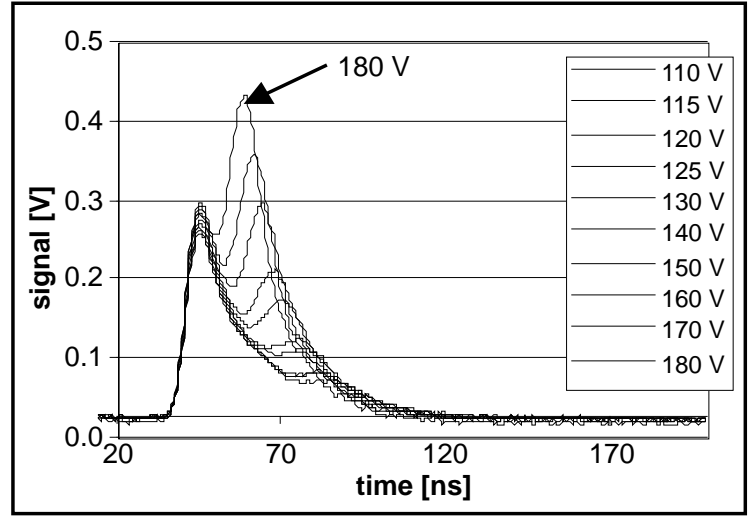

(b)

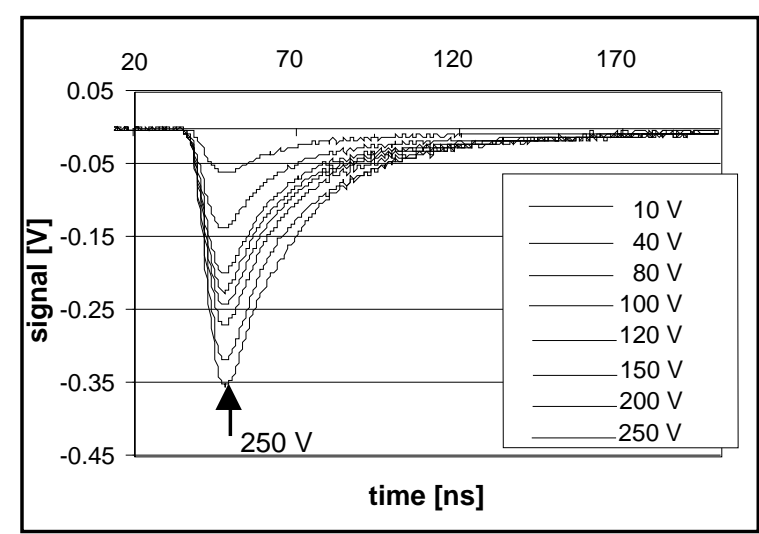

(d)

Fig. 9: Signal pulse shape for diode irradiated up to $2.9 \times 10^{14} \mathrm{~cm}^{-2}$ as a function of bias (a) (b) (c).

Front illumination. (d) Rear illumination. $V_{\mathrm{FD}}=225 \mathrm{~V}$

These experimental results can be explained with the hypothesis of a depleted region on each side of the detector and with an electric field distribution qualitatively shown in Fig. 10, as proposed by the Lancaster group [6]. A weak electric field should be able to drive the charge in 
the undepleted volume between the two depleted regions. The movement of the charge (electrons) in the depleted region next to the front side junction, in the direction of a decreasing field, generates the first peak. The electrons, passing through the intermediate undepleted region, experience a trapping process. When the undepleted volume narrows enough to permit the electrons to reach the depleted volume next to the main rear-side junction, the surviving electrons move in the sensitive volume up to the $\mathrm{n}^{+}$contact, inducing the second peak signal.

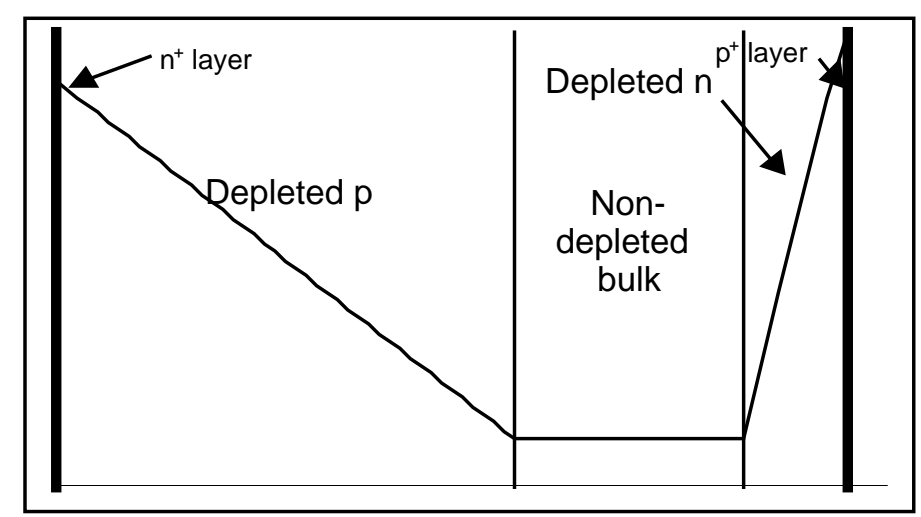

Fig. 10: Qualitative shape of the electric field in an inverted irradiated silicon detector [6]

The time dependence of the second peak as a function of the square root of bias is shown in Fig. 11. The time of the peak position seems to decrease proportionally to $V^{1 / 2}$ for bias voltages lower then $V_{\mathrm{FD}}$. This effect is mainly due to the travel time in the non-depleted region. Below full depletion, the peak position time, $t_{\mathrm{p}}$, can be expressed by

$$
t_{\mathrm{p}}-t_{\mathrm{p}, \mathrm{FD}}=\frac{1}{v}(w-x)=\frac{w}{v}\left(1-\frac{\sqrt{V}}{\sqrt{V_{\mathrm{FD}}}}\right),
$$

where $t_{\mathrm{p}, \mathrm{FD}}$ is the time of the peak position at full depletion, $v$ the velocity of the carriers, $w$ the detector thickness, and $x$ the depleted volume depth. For bias voltages below full depletion, $t_{\mathrm{p}}$ increases linearly with $V^{1 / 2}$, indicating the independence of the velocity on the applied bias. The fit of the experimental data with Eq. (5) allows the evaluation of the velocity. The strength of the electric field in the undepleted bulk, $E_{0}$, is calculated using $E_{0}=\nu / \mu$. The mobility value, $\mu$, used here is $1100 \mathrm{~V}^{-1} \mathrm{~cm}^{2} \mathrm{~s}^{-1}$ [7]. The values of $v$ and $E_{0}$ are reported in Table 2 for three different irradiation levels.

Table 2: Values of the velocity and the electric field in the non-depleted bulk

\begin{tabular}{|c|c|c|}
\hline Fluence $\left[\mathrm{cm}^{-2}\right]$ & Velocity $\left[\mathrm{cm} \mathrm{s}^{-1}\right]$ & $E_{0}\left[\mathrm{~V} \mathrm{~cm}{ }^{-1}\right]$ \\
\hline $1 \times 10^{14}$ & $1.35 \times 10^{5}$ & 123 \\
$1.7 \times 10^{14}$ & $1.45 \times 10^{5}$ & 132 \\
$2.9 \times 10^{14}$ & $2.31 \times 10^{5}$ & 210 \\
\hline
\end{tabular}




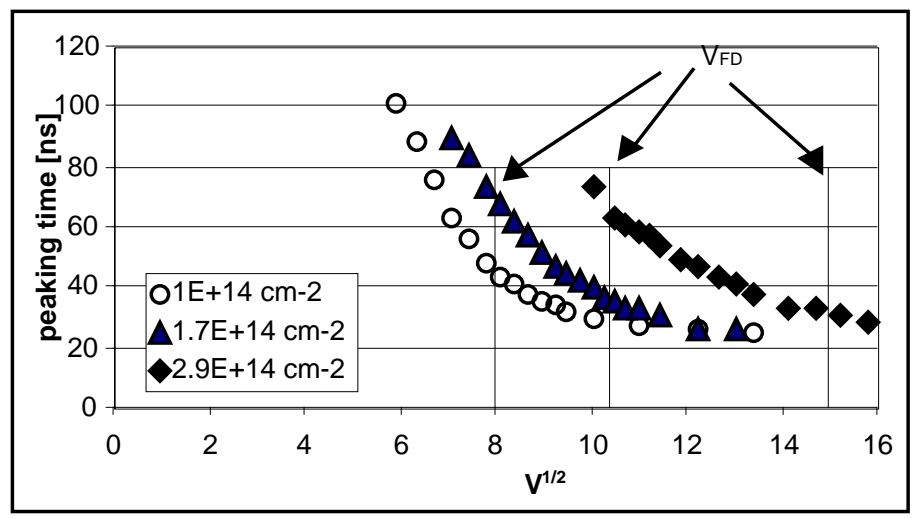

Fig. 11: Time dependence of the second peak as a function of $V^{1 / 2}$

The charge collection efficiency curves as a function of $V^{1 / 2}$ for front illumination of the inverted diodes (Fig. 6b) can be divided into three parts. At lower biases there is a linear dependence on $V^{1 / 2}$ due to the contribution of the first peak only. Increasing the bias, the contribution of the first peak to the collected charge saturates and the second peak appears and contributes to the collected charge. The contribution of this peak is not linear with $V^{1 / 2}$ because of the trapping experienced by the charge traversing the undepleted bulk. The CCE as a function of the bias in the interval from the appearance of the second peak contribution, $V_{0}$, to $V_{\mathrm{FD}}$ can be expressed as follow

$$
\mathrm{CCE}=C_{1}+C_{2} \sqrt{V} \mathrm{e}^{-\underline{\underline{x}}}
$$

where

$$
x=w-d=w\left(1-\frac{\sqrt{V}}{\sqrt{V_{\mathrm{FD}}}}\right)
$$

is the thickness of the undepleted bulk. The constans $C_{1}$ and $C_{2}$ can be found using the boundary conditions $\mathrm{CCE}=C_{0}$ for $V=V_{0}$ and $\mathrm{CCE}=E_{\mathrm{FD}}$ for $V=V_{\mathrm{FD}} . E_{\mathrm{FD}}<1$ in irradiated diodes because of the trapping effect. $E_{\mathrm{FD}}, C_{0}$ and $V_{0}$ have to be experimentally evaluated for any fluence. The expressions for $C_{1}$ and $C_{2}$ are then

$$
\begin{gathered}
C_{1}=E_{\mathrm{FD}}-C_{2} \sqrt{V_{\mathrm{FD}}} \\
C_{2}=\frac{\left(E_{\mathrm{FD}}-C_{0}\right)}{\sqrt{V_{\mathrm{FD}}} \mid 1-y e^{\left.-\frac{w}{a}(1-y)\right)},},
\end{gathered}
$$

where $y=\sqrt{ } V_{0} / N_{\mathrm{FD}}$. The parameter $a$ defines the attenuation length of the electrons in the undepleted bulk. The fits to the CCE curves in Fig. $6 \mathrm{~b}$ give values of $a$ between 70 to $90 \mu \mathrm{m}$ for the heavily irradiated diodes. After full depletion, the CCE increases as a function of the applied reverse bias due to the decreased effectiveness of trapping. 


\section{DETECTORS AROUND THE TYPE-INVERSION FLUENCE}

For type-inverted detectors the double peak is only visible when the diode is illuminated on the front side. The electric field distribution shown in Fig. 10 predicts a double peak also with rear injection. In this case, in a partially depleted detector, the holes generated from the light at the rear surface drift towards the anode in the depleted rear-side volume. The charge reaches the undepleted bulk, where it experiences trapping, and the surviving carriers induce a second signal moving in the depleted front-side bulk. The situation is symmetric to the signal induced by electrons in the case of front illumination. The absence of the second peak and the linearity of the CCE as a function of $V^{1 / 2}$ indicate that the signal caused by the holes moving in the front-side depleted bulk is negligible. This can be explained by the more effective trapping of holes in the undepleted bulk, because of the lower mobility of the holes, and by the smaller depth of the front-side depletion compared to the rear-side. For irradiated detectors close to the type inversion fluence, the double peak in the signal is visible for illumination from both sides, as shown in Figs. 12a and $12 \mathrm{~b}$ for a diode irradiated up to $5.2 \times 10^{13} \mathrm{~cm}^{-2}$. The contribution of the second peak appears in both cases at $\approx 15 \mathrm{~V}$. The peak time evolution as a function of $V^{1 / 2}$ is shown in Fig. 13. The difference between the front and the rear injection is due to the different mobility of electrons and holes.

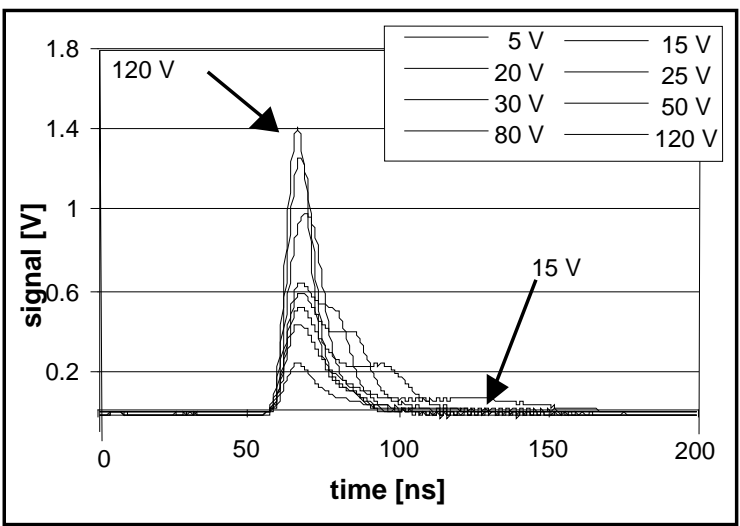

(a)

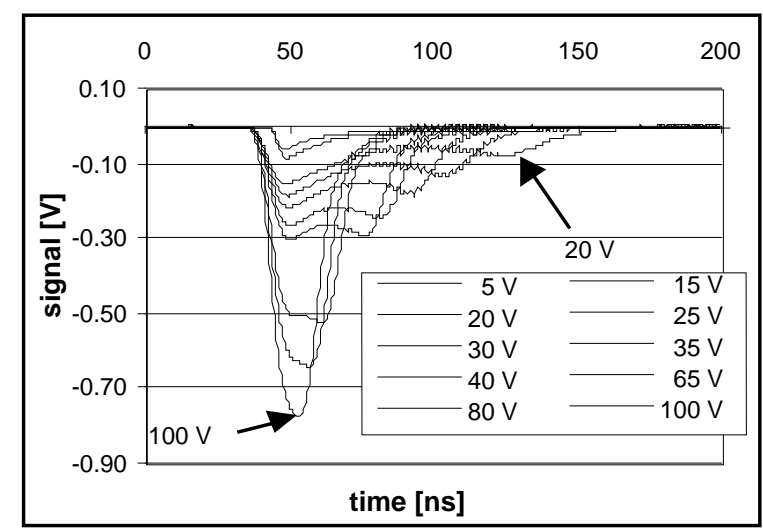

(b)

Fig. 12: Signal pulse shape for a diode irradiated up to $5.2 \times 10^{13} \mathrm{~cm}^{-2}$

(a) Front illumination (b) Rear illumination. $V_{\mathrm{FD}}=25 \mathrm{~V}$

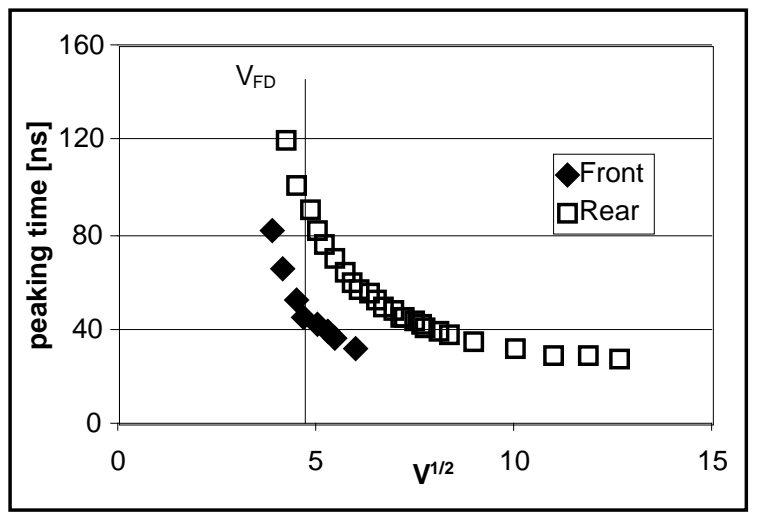

Fig. 13: Peaking time as a function of $V^{1 / 2}$ for front-and rear- side injection in a diode irradiated up to 


$$
5.2 \times 10^{13} \mathrm{~cm}^{-2}
$$

\section{DISCUSSION}

This study has confirmed the presence of strong electric fields and two depleted regions on both sides of an irradiated detector. The irradiated detectors behave as with a double-junction. The charge collection starts from low biases for front- or rear-side illumination. The existence of a weak electric field able to drive the generated charge in the undepleted bulk that separates the two sensitive regions of an irradiated detector has been established. The strength of this electric field has been evaluated for three high proton fluences, after detector conductivity-type inversion.

A model is proposed to interpret the charge collection property of diodes irradiated above type-inversion fluence and to fit the CCE characteristic as a function of $V^{1 / 2}$. A partially-depleted irradiated diode has two sensitive regions close to the contacts separated from a quasi-neutral undepleted bulk. This model assumes a linear dependence of the CCE on the square root of bias when the charge moves in the depleted bulk, and accounts for the trapping of the carriers moving in the undepleted bulk. The results of the fit seem to confirm the validity of the assumption. It can be observed, from the signal shape and from the charge collection properties, that the front-side depleted volume of the type inverted detectors is smaller compared to the rear-side depleted volume. After type-inversion, the dominating junction is on the rear-side. Around type-inversion, a comparable signal is visible on both sides of the detector, demonstrating that the extension of the depleted volume as a function of bias is comparable on both sides. This suggests that even before type-inversion, the decrease of $N_{\text {eff }}$ is accompanied by the build-up of a junction in the rear side of the detectors. This hypothesis is supported by simulation results of the silicon detector changes as a function of fluence using the introduction of two deep defect levels in the forbidden band, one acting as an acceptor and one as a donor dopant [8].

\section{REFERENCES}

[1] E. Gatti and P.F. Manfredi, Riv. Nuovo Cimento 9, No.1 (1986).

[2] F. Lemeilleur et al., Proc. $1^{\text {st }}$ Int. Conf. on large scale applications and radiation Hardness of Semiconductor Detectors, Firenze, 1993, Vol. 46 167-180.

[3] H.W. Kraner et al., Nucl. Instrum. and Methods A326 (1993) 350-256.

[4] F. Lemeilleur et al., IEEE Trans. Nucl. Sci. Vol. 39 (1992) 551.

[5] Z. Li and E. W. Kraner, IEEE Trans. Nucl. Sci. 38 (1991) 244.

[6] L.J. Beattie et al., ATLAS note INDET-Nº 194, 10 December 1997.

[7] C. Leroy et al., Proc. $2^{\text {nd }}$ Int. Conf. on radiation effects on semiconductor materials: detectors and devices, Firenze, Italy, 1998, to be published in Nucl. Instrum. and Methods.

[8] D. Passeri et al., Proc. $2^{\text {nd }}$ Int. Conf. on radiation effects on semiconductor materials, detectors and devices, Firenze, Italy, 1998, to be published in Nucl. Instrum. and Methods. 


\section{APPENDIX A}

\section{A1. SIGNAL FORMATION IN A SILICON DETECTOR}

The passage of an ionizing particle through a detector generates electron-hole $(\mathrm{e}-\mathrm{h})$ pairs. The electric field in the depletion region drifts electrons towards the reverse biased $\mathrm{n}^{+}$contact. Similarly, holes drift towards the $\mathrm{p}^{+}$contact. The induced charge then produces a signal on the detector electrodes according to Ramo's theorem

$$
\Delta Q=Q \frac{\Delta x}{w},
$$

where $w$ is the detector thickness. The induced current is given by

$$
\mathrm{i}(\mathrm{x})=\frac{\mathrm{dQ}}{\mathrm{dt}}=\frac{\mathrm{Q}}{\mathrm{w}} \frac{\mathrm{dx}}{\mathrm{dt}}=\frac{\mathrm{Q}}{\mathrm{w}} \mu \mathrm{E}(\mathrm{x}) .
$$

The electric field inside a detector is expressed by

$$
\begin{array}{rr}
E(x)=-\frac{q N_{\mathrm{eff}}}{\varepsilon_{S i}}(x-w)=-a x+b & V=V_{\mathrm{FD}} \\
E(x)=-\frac{q N_{\mathrm{eff}}}{\varepsilon_{S i}}(x-w)+\frac{V-V_{\mathrm{FD}}}{w}=-a x+b & V=V_{\mathrm{FD}}
\end{array}
$$

where $V_{\mathrm{FD}}$ is the full depletion voltage. The constant $b$ expresses the maximum value of the electric field at the junction. The electric field is directed in the negative direction of the $x$-axis. The holes drift towards the reverse biased $\mathrm{p}^{+}$contact and the electrons drift in the opposite direction. The drift velocity of the electrons and holes can be expressed as follows:

$$
\begin{aligned}
\frac{d x_{e}}{d t} & =-\mu_{e}(a x-b) \\
\frac{\mathrm{d} x_{h}}{\mathrm{~d} t} & =-\mu_{h}(a x-b),
\end{aligned}
$$

where $\mu_{e, h}$ is the mobility of electrons or holes. Integrating Eq. (A.5) for a unit charge generated in $x=x_{0}$ gives, for electrons and holes, respectively

$$
\begin{aligned}
& x_{e}(t)=\frac{b}{a}+\left(x_{0}-\frac{b}{a}\right) \exp \left(-\mu_{e} a t\right) \\
& x_{h}(t)=\frac{b}{a}+\left(x_{0}-\frac{b}{a}\right) \exp \left(\mu_{h} a t\right) .
\end{aligned}
$$

The velocity is then

$$
\begin{gathered}
v_{e}(t)=-\mu_{e}\left(a x_{0}-b\right) \exp \left(-\mu_{e} a t\right) \\
v_{h}(t)=-\mu_{h}\left(a x_{0}-b\right) \exp \left(-\mu_{h} a t\right) .
\end{gathered}
$$


We can define the collection time $T_{\text {ce }}$ for electrons and $T_{\text {ch }}$ for holes as follows

$$
\begin{aligned}
& T_{\mathrm{ce}}=\frac{1}{a \mu_{e}} \ln \left(\frac{a x_{0}-b}{a w-b}\right), x\left(T_{\mathrm{ce}}\right)=w \\
& T_{\mathrm{ch}}=\frac{1}{a \mu_{h}} \ln \left(\frac{b}{b-a x_{0}}\right), x\left(T_{\mathrm{ch}}\right)=0,
\end{aligned}
$$

The current induced by electrons and holes separately, as a function of time, is given by

$$
\begin{array}{cc}
i_{\mathrm{e}}(t)=\frac{q \mu_{e}}{w}\left(a x_{0}-b\right) \exp \left(-\mu_{\mathrm{e}} a t\right) & 0 \leq t \leq T_{\mathrm{ce}} \\
i_{\mathrm{h}}(t)=\frac{q \mu_{h}}{w}\left(a x_{0}-b\right) \exp \left(-\mu_{\mathrm{h}} a t\right) & 0 \leq t \leq T_{\mathrm{ch}} .
\end{array}
$$

The observable signal is the convolution of the currents produced by all the generated charges. The signal caused by holes increases with time and the signal caused by electrons decreases with time. The charge collected is evaluated by the integration over time of the hole and electron currents

$$
\begin{array}{cc}
Q_{\mathrm{e}}(t)=\frac{-q}{a w}\left(a x_{0}-b\right)\left[\exp \left(-\mu_{\mathrm{e}} a t\right)-1\right] & 0 \leq t \leq T_{\mathrm{ce}} \\
Q_{\mathrm{h}}(t)=\frac{q}{a w}\left(a x_{0}-b\right)\left[\exp \left(-\mu_{\mathrm{e}} a t\right)-1\right] & 0 \leq t \leq T_{\mathrm{ch}} \\
Q_{\text {etot }}=\frac{q}{w}\left(w-x_{0}\right) & \\
Q_{\mathrm{tot}}=\frac{q}{w} x_{0} . &
\end{array}
$$

The sum of both of the contributions gives the unit charge injected.

\section{A2. TRAPPING EFFECT}

When a detector is over-depleted, all the charge is created in the depletion region of the detector. The integral of the $I(t)$ signal provides the total charge deposited from the incident ionizing particle.

$$
Q_{\mathrm{dep}}=\int_{0}^{t_{\mathrm{end}}} I(t) \mathrm{d} t .
$$

This is the case when the generated charge can drift towards the electrodes without any trapping. If trapping centres for both types of carrier are present, they can reduce the total charge collected at the electrodes. To take into account this effect we introduce the characteristic trapping $\left(\tau_{\mathrm{t}}\right)$ and detrapping $\left(\tau_{\mathrm{d}}\right)$ lifetimes. Normally $\tau_{\mathrm{d}} \gg \tau_{\mathrm{t}}$ and the detrapping effect can be neglected. If the trap concentration is uniform and large compared to the created charge, the reduced number of charges arriving at the electrodes can be expressed by 


$$
n_{0}(t)=n_{0} \exp \left(-\frac{t}{\tau_{\mathrm{t}}}\right)
$$

The trapping lifetime is defined as

$$
\tau_{\mathrm{t}}=\frac{1}{\sigma_{1} V_{\mathrm{th}} N_{\mathrm{T}}}
$$

where $\sigma_{t}$ is the trapping cross-section, $v_{\mathrm{th}}$ the thermal velocity, and $N_{\mathrm{T}}$ the trap concentration. 